\title{
Índices de Eficiência do Distrito de Irrigação "Sector BXII del Bajo Guadalquivir", Sul da Espanha, por Sensoriamento Remoto
}

Conference Paper · January 2014

DOI: 10.12702/ii.inovagri.2014-a237

READS

20

3 authors, including:

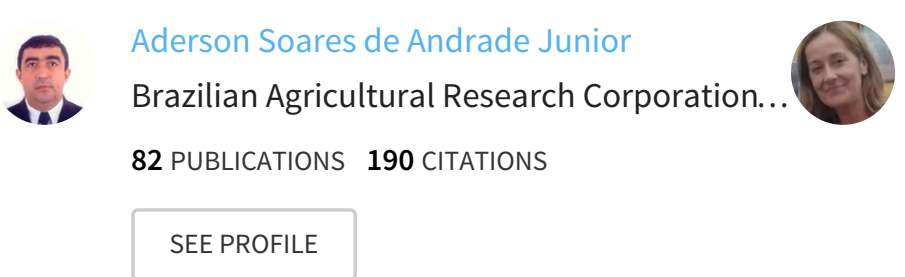

María P. González-Dugo

Instituto de Investigación y Formación Agra...

39 PUBLICATIONS 580 CITATIONS

SEE PROFILE 


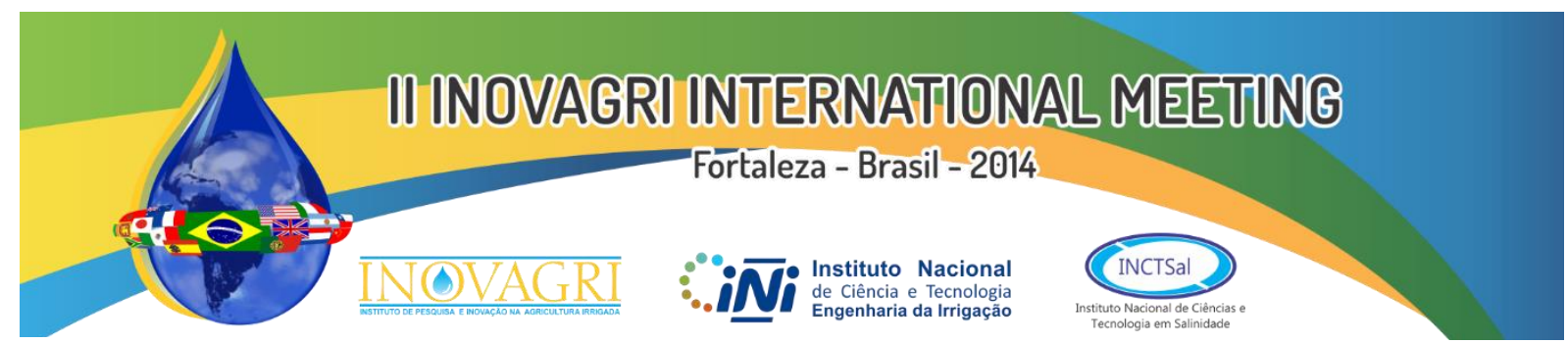

http://dx.doi.org/10.12702/ii.inovagri.2014-a237

\title{
INDICES DE EFICIÊNCIA DO DISTRITO DE IRRIGAÇÃO "SECTOR BXII DEL BAJO GUADALQUIVIR”, SUL DA ESPANHA, POR SENSORIAMENTO REMOTO
}

\author{
A.S. ANDRADE JUNIOR ${ }^{1}$, L. MATEOS $^{2}$, M.P. GONZALEZ-DUGO ${ }^{3}$
}

\begin{abstract}
RESUMO: A avaliação do desempenho dos distritos de irrigação é estratégica para a melhoria da qualidade da gestão da água em grandes áreas ou bacias hidrográficas. Há diversos índices de eficiência, dentre estes se destacam evapotranspiração relativa - RET; coeficiente de déficit hídrico - CDH; índice de uso consumptivo - ICUC; suprimento relativo da irrigação - RIS e suprimento relativo de água (chuva mais irrigação) - RWS e de produtividade da água - WP. O estudo teve como objetivo avaliar o desempenho do distrito de irrigação "Sector BXII del Bajo Guadalquivir", região Sul da Espanha, por meio da definição de índices de eficiência e produtividade da água de irrigação. Procedeu-se a estimativa da evapotranspiração ET dos cultivos por meio de índices de vegetação (Soil Adjusted Vegetation Index SAVI), com o uso da ferramenta do sensoriamento remoto (Landsat TM) e simulou-se a adoção do coeficiente de cultivo (Kc) dual da FAO (Kcb-Savi) em comparação ao Kc sintético (Kcs), ambos usando o plano de cultivo fornecido pela comunidade dos irrigantes. O emprego do Kcb-Savi permitiu o alcance de índices de eficiência de aplicação de água satisfatórios (RET $=0,778, \mathrm{CDH}=0,222, \mathrm{ICUC}=0,922, \mathrm{RIS}=$ 1,028 e RWS $=0,949$ ). A adoção do Kcs possibilitou a estimativa dos índices de eficiência sem diferenças significativas em relação ao emprego do Kcb-Savi $(\mathrm{RET}=0,783, \mathrm{CDH}=0,217$, ICUC $=1,037$, RIS $=$ $1,028$ e RWS $=0,944)$. O cultivo do tomate $\left(18,05 \mathrm{~kg} / \mathrm{m}^{3}\right)$ apresentou produtividade da água de irrigação superior aos cultivos do algodão $\left(0,66 \mathrm{~kg} / \mathrm{m}^{3}\right)$ e beterraba $\left(10,43 \mathrm{~kg} / \mathrm{m}^{3}\right)$, devido ao melhor manejo da água de irrigação efetuado pelos produtores de tomate.
\end{abstract}

PALAVRAS-CHAVE: gestão de água, planejamento de irrigação, índices de vegetação.

\section{EFFICIENCY INDEXES OF "SECTOR BXII DEL BAJO GUADALQUIVIR" IRRIGATION DISTRICT, SOUTHERN OF SPAIN, USING REMOTE SENSING}

SUMMARY: The performance evaluation of irrigation districts is critical to improving water management in large areas or watersheds. There are several irrigation efficiency indexes, such as relative evapotranspiration - RET; coefficient of water deficit - CWD; index consumptive use - ICUC; relative irrigation supply - RIS and relative water supply (rain plus irrigation) - RWS and water productivity - WP were evaluated. This study aimed to evaluate the performance of the "Sector BXII del Bajo Guadalquivir" Irrigation District, the southern region of Spain, through the definition of indexes of efficiency and irrigation water productivity. Therefore, proceeded to estimate the crop evapotranspiration (ET) through vegetation indices (Soil Adjusted Vegetation Index - SAVI), using remote sensing tool (Landsat TM) and dual crop coefficient (Kc) FAO (Kcb-Savi) in compared to synthetic Kc (Kcs) were simulated, both using the crop plan provided by the irrigation community. The employment of Kcb-Savi allowed the achievement of efficiency rates of water application satisfactory $(\mathrm{RET}=0.778, \mathrm{CWD}=0.222$, ICUC $=0.922$, RIS $=$ 1.028 and RWS $=0.949)$. The use of Kcs allowed for the estimation of the efficiency ratios with no significant differences in relation to the employment of Kcb-Savi $(\mathrm{RET}=0.783, \mathrm{CWD}=0.217$, ICUC $=$ 1.037 , RIS $=1.028$ and RWS $=0.944)$. Tomato $\left(18.05 \mathrm{~kg} / \mathrm{m}^{3}\right)$ showed productivity of irrigation water above of cotton $\left(0.66 \mathrm{~kg} / \mathrm{m}^{3}\right)$ and sugar beet $\left(10.43 \mathrm{~kg} / \mathrm{m}^{3}\right) \mathrm{crops}$, due to better management irrigation water made by tomato growers.

\footnotetext{
${ }^{1}$ Pesquisador, Embrapa Meio-Norte, Av. Duque de Caxias, 5650, CEP 64006-220, Teresina, Piauí. Fone: (86) 3089-9160. E.mail: aderson.andrade@embrapa.br

${ }^{2}$ Pesquisador, Instituto de Agricultura Sostenible (IAS), Córdoba, Espanha.

${ }^{3}$ Pesquisadora, Instituto de Investigación y Formación Agraria y Pesquera (IFAPA), Córdoba, Espanha.
} 
KEYWORDS: water management, irrigation planning, vegetation index.

\section{INTRODUÇÃO}

Em termos mundiais, a água destinada para fins agrícolas $\left(2664 \mathrm{~km}^{3}\right)$ representa $70 \%$ da água disponível para usos múltiplos $\left(3830 \mathrm{~km}^{3}\right)(\mathrm{FAO}, 2007)$. Na Espanha, a demanda por água doce é estimada em 35 a $105 \mathrm{~m}^{3} /$ ano, com cerca de $70 \%$ dedicado a irrigação e o restante para outros usos. Nos próximos 10 anos, o governo espanhol prevê um aumento de $17 \%$ na demanda de água para fins de irrigação no sul da Espanha (MIMAM, 2000). Por isso, a avaliação do desempenho dos distritos de irrigação é o ponto de partida para a melhoria da gestão da água em grandes áreas ou bacias hidrográficas (Lorite et al., 2004a).

O desempenho dos distritos de irrigação é mensurado por meio de inúmeros índices de eficiência e de produtividade da água (Bos et al., 2005). Porém, para que essa mensuração seja efetuada com melhor acurácia, é necessária a disponibilidade de registros dos volumes de água efetivamente aplicados aos cultivos por meio da irrigação, a fim de permitir a estimativa adequada dos demais componentes do balanço hídrico em campo, bem como para conhecer o padrão de aplicação de água ao nível de cada parcela (Buller et al., 1991; Heinemann et al., 2002; Lorite et al., 2004a).

A quantificação da evapotranspiração dos cultivos (ETc) é de fundamental importância para que seja possível a estimativa dos balanços hídricos e dos índices de eficiência de irrigação. A ETc é obtida pelo produto da evapotranspiração de referencia (ETo) pelo coeficiente de cultivo (Kc) apropriados a cada fase de desenvolvimento (Allen et al., 1998). A ETo é estimada com base em modelos que utilizam variáveis climáticas diárias obtidas de estações meteorológicas, sendo o de Penman-Monteith, o modelo padrão recomendado pela FAO (Allen et al., 1998). Porém, para que a estimativa de ETc seja aplicada a grandes áreas irrigadas, torna-se necessário que a estimativa do Kc seja espacialmente distribuída, por meio de índices de vegetação multiespectrais (IVs), que são estimados a partir das reflectâncias espectrais da superfície medidas em bandas específicas do espectro eletromagnético, tipicamente, o vermelho e o infravermelho próximo, obtidos por sensoriamento remoto (Bausch \& Neale, 1987; Neale et al., 1989; Calera et al., 2004; Gonzalez-Dugo \& Mateos, 2008; Campos et al., 2010; Glenn et al., 2011), tais como o "Normalized Difference Vegetation Index" (NDVI) (Rouse et al., 1974) e "Soil Adjusted Vegetation Index" (SAVI) (Huete, 1988).

Mateos et al. (2013) propôs um método alternativo para estimativa de ET-IVs usando o conceito de Kc simplificado (Kc sintético), que possui maior potencial de aplicação em larga escala. A relação entre Kcb e Kcs foi estudada usando dados diários de um balanço hídrico diário descrito em GonzalezDugo et al. (2009) e validado com os dados de ET de vários cultivos anuais (alho e algodão) e arbóreos (mandarina, olivo e pêssego) obtidos por Eddy covariância. Os resultados mostraram haver um bom ajuste entre as duas metodologias de estimativa, com resultados promissores para aplicação em grandes áreas irrigadas ou bacias hidrográficas (Gonzalez-Dugo et al., 2013).

O estudo tem como objetivo avaliar o desempenho do distrito de irrigação "Sector B-XII del Bajo Guadalquivir" por meio da definição de índices de eficiência e produtividade da água de irrigação. Para tanto, propõe-se a estimativa da ET dos cultivos com o conceito do coeficiente de cultivo dual (Kcb-Savi) em comparação ao coeficiente de cultivo sintético (Kcs), ambos associados ao uso do plano de cultivo da comunidade dos irrigantes, com o intuito de facilitar a operacionalização da estimativa desses índices por meio do uso da ferramenta do sensoriamento remoto.

\section{MATERIAL E MÉTODOS}

O distrito de irrigação "Sector B-XII del Bajo Guadalquivir" está situado em Lebrija, província de Sevilha, comunidade autônoma da Andaluzia, região Sudeste da Espanha, com as seguintes coordenadas em seu ponto central: $36^{\circ} 58^{\prime} 26^{\prime \prime} \mathrm{N}, 06^{\circ} 07^{\prime} 15^{\prime \prime}$ ' O e $2 \mathrm{~m}$ de altitude (Figura 1).

O fornecimento de água é realizado a partir de um reservatório situado no trecho final do "Canal del Bajo Guadalquivir", por meio de três canais secundários, os quais distribuem água para três setores (A, B e C), que compõem o distrito. A vazão do canal principal é de $15 \mathrm{~m}^{3} / \mathrm{s}$, a qual é subdividida em $5,65,5,40$ e $3,45 \mathrm{~m}^{3} / \mathrm{s}$ nos canais secundários que distribuem água aos setores $\mathrm{A}, \mathrm{B}$ e $\mathrm{C}$, 


\section{A. S. Andrade Junior et al.}

respectivamente (Lozano \& Mateos, 2008). A distribuição de água as parcelas dos irrigantes é efetuada por bombas distribuídas ao longo dos canais secundários (Figura 1).

$\mathrm{O}$ distrito tem uma área total de aproximadamente 14.000 ha, distribuídos em 1.170 parcelas, destinados, notadamente, aos cultivos de algodão (44,98\%), beterraba açucareira $(22,71 \%)$, tomates (11,65\%), girassol (4,21\%), milho (4,18\%) e trigo (4,73\%), que cujas áreas de cultivo totalizam $96,3 \%$ da área. O clima é Mediterrâneo, com verão quente e seco e inverno/outono chuvoso e úmido. O solo foi originado dos sedimentos finos estuarinos do rio Guadalquivir, com elevado teor de argila e baixo conteúdo de areia (Lozano \& Mateos, 2008).

Os dados básicos para a execução do estudo consistiram do plano de cultivos e dos volumes diários de água aplicados às parcelas do distrito de irrigação "Sector B-XII del Bajo Guadalquivir", chamado abreviadamente de "B-XII", referentes ao ano agrícola de 2011. Para tanto, fez-se uso do software ArcGIS e planilha eletrônica Excel. Elegeram-se para avaliação os cultivos de algodão, girassol, milho, beterraba açucareira, tomates e trigo, já que representam 96,3\% da área cultivada na campanha de 2011. Esses cultivos são explorados, notadamente, em sistema de múltiplos cultivos (10791,0 ha), com 89,4\%, e, em menor proporção, na forma de cultivos puros (1280,1 ha), com 10,6\% da área cultivada. As principais associações de cultivo envolvem algodão, beterraba e tomates.

O plano de cultivo foi fornecido pela gerência do distrito de irrigação, indicando o cultivo, a área ocupada e sua posição em cada parcela. Esses dados foram georreferenciados com o software ArcGIS 9.3 (ESRI, 2008), o que permitiu geoespacializar essa informação em campo. Posteriormente, após o geoprocessamento das imagens de satélite e a estimativa do índice de vegetação Savi, usandose a variação temporal desse índice ao longo da estação de cultivo, o plano de cultivo repassado foi validado e ajustado, corrigindo-se, caso necessário, eventuais discrepâncias entre ambos. Os volumes diários de água aplicados em cada parcela foram fornecidos pela gerência do distrito de irrigação. Esses volumes são oriundos de leituras dos hidrômetros posicionados na entrada de cada parcela, registrados durante o período de janeiro a novembro de 2011.

Para as parcelas com cultivos de algodão, beterraba açucareira e tomate também foram obtidos, junto às cooperativas dos produtores, os dados de produção total por parcela do ano agrícola de 2011. Esses dados de produção e de água aplicada as parcelas são essenciais para a estimativa dos índices de eficiência e de produtividade da água, conforme será detalhado adiante.

\section{$\underline{\text { Tratamento das imagens de satélite }}$}

Utilizou-se um conjunto com 14 imagens de satélite Landsat 5 TM (cinco imagens) e Landsat 7 $\mathrm{ETM}+$ (nove imagens) obtidas durante o ano de 2011, referente a cena 202/34, que abrange a área do distrito de irrigação "Sector B-XII". A distribuição das datas com disponibilidade de imagens por cada sensor foi a seguinte: Landsat 5 TM (09/02/11, 25/02/11, 01/06/11, 04/08/11 e 08/11/11) e Landsat 7 ETM+ (01/02/11, 21/03/11, 06/04/11, 08/05/11, 24/05/11, 28/08/11, 13/09/11, 15/10/11 e 31/10/11). As imagens foram obtidas gratuitamente por meio da internet no site do USGS - United States Geological Survey (http://glovis.usgs.gov/).

O procedimento de geoprocessamento das imagens constou das etapas de correção geográfica e atmosférica de todas as bandas, conforme procedimento descrito em Gonzalez-Dugo et al. (2009, 2013). Para a correção atmosférica foi aplicado o módulo FLAASH (Fast Line-of-Sight Atmospheric Analysis of Spectral Hypercube) (Adler-Golden et al., 1999), baseado em um modelo de transferência radioativa MODTRAN4 (Berk et al. 1998), associado a correção de aerossóis e vapor de água na atmosfera com o modelo 6S (Vermote et al., 1997). Todo esse procedimento foi executado no software ENVI, versão 4.7 (ITT, 2009).

\section{Estimativa de índices de vegetação / ET dos cultivos}

Procedeu-se a estimativa do índice de vegetação SAVI ("Soil Adjusted Vegetation Index") (Huete, 1988), conforme descrito em Gonzalez-Dugo \& Mateos (2008). Esse índice é a base para a estimativa da ET dos cultivos por teledetecção.

A estimativa da evapotranspiração dos cultivos (ETc) foi efetuada por meio da aplicação de um modelo de balanço de água no solo, conforme metodologia proposta pela FAO (Allen et al., 1998), 
descrita em Lorite et al. (2004a, 2007). Para tanto, usou-se uma planilha em Excel associada à ferramenta de macros, para automatizar a geração dos balanços hídricos de todas as parcelas avaliadas.

No processamento dos balanços hídricos e estimativa da ETc foram admitidas duas situações: i) uso do coeficiente de cultivo dual (Kcb), com base no índice de vegetação Savi e fração de cobertura máxima do solo $(\mathrm{Fc})$, conforme apresentado em Gonzalez-Dugo \& Mateos (2008) e Gonzalez-Dugo et al. (2009), identificado como Kcb-Savi e ii) uso do coeficiente de cultivo sintético (Kcs), conforme apresentado em Mateos et al. (2013), intitulado como Kcs:

Os valores de Kcb e Kc máximos dos cultivos foram obtidos de Allen et al. (1998). Processouse, adicionalmente, os balanços hídricos com o uso do coeficiente de cultivo dual padrão da FAO (Allen et al., 1998), denominada de Kcb-FAO, com os quais obteve-se os valores de evapotranspiração potencial para cada cultivo (ETp), para aplicação na estimativa dos índices de eficiência de irrigação.

Os dados climáticos diários de precipitação e evapotranspiração de referencia (ETo), estimada por Penman -Monteith FAO (Allen et al., 1998), do ano de 2011, necessários ao modelo de balanço de água no solo, foram obtidos da estação Lebrija I, que pertence a rede de estações agrometeorológicas do Instituto de Investigação Agraria e Pesqueira - IFAPA, disponibilizados via internet no site http://www.juntadeandalucia.es/agriculturaypesca/ifapa/ria. Essa estação situa-se em uma zona muito próxima do "Sector B-XII" (Latitude: 36 58 40 " N, Longitude: 0607'30" W e altitude de $25 \mathrm{~m}$ ).

A ET obtida dos balanços hídricos correspondeu ao somatório da ETc diária de cada cultivo ocorrida no período semeadura - colheita. Considerou-se que, para as parcelas com múltiplos cultivos, a ET da parcela como a soma da ET de cada um dos cultivos. Esse procedimento foi necessário, já que os índices de eficiência e de produtividade da água foram estimados para cada parcela e não para cada cultivo dentro da parcela. Para cada condição avaliada, os valores de ETc obtidos foram utilizados na estimativa dos índices de eficiência e de produtividade da água, permitindo avaliar o impacto da adoção de cada valor de coeficiente de cultivo (Kc) na estimativa desses índices.

\section{Estimativa de índices de eficiência e produtividade da água}

Para a estimativa da eficiência e produtividade da água de irrigação utilizou-se os seguintes índices: a) índice de evapotranspiração relativa (RET), coeficiente de deficiência hídrica (CDH), índice de uso consumptivo da água de irrigação (ICUC), índice de suprimento relativo de irrigação (RIS), índice de suprimento relativo de água (irrigação mais chuva) (RWS) e índice de produtividade da água evapotranspirada pelos cultivos (WP) (Bos et al., 2005).

Para a estimativa dos índices de eficiência usando o Kcs, para simplificar a aplicação do método, considerou-se a precipitação efetiva $(\mathrm{Re})$ igual a aplicada ao cálculo dos índices de eficiência com o uso de Kcb-Savi. Da mesma forma, admitiu-se a variação do armazenamento de água no solo como um valor fixo e igual à média da variação do armazenamento advinda dos balanços hídricos com o uso do Kcb-Savi $(15 \mathrm{~mm})$. Tendo em vista que a comparação pretendida é aplicada à mesma área (mesmo solo), essa simplificação não interferiu de maneira decisiva na estimativa dos índices de eficiência com o uso do Kcb, conforme será demonstrado na discussão dos resultados.

A estimativa do índice WP foi efetuada apenas com dados de produção de parcelas com cultivos de algodão, beterraba e tomates, cujos produtores estão mais organizados e direcionam a produção às suas respectivas cooperativas, as quais nos forneceram os dados de produção obtidos no ano de 2011.

\section{RESULTADOS E DISCUSSÃO}

$\underline{\text { Volumes de água aplicados às parcelas }}$

Os volumes totais mensais de água aplicados às parcelas por estação de bombeamento e setor, durante o período de janeiro a setembro de 2011, são apresentados na Tabela 1. A distribuição percentual mensal do volume total aplicado, durante o período de janeiro a setembro de 2011, é apresentada na Figura 2.

O volume total aplicado, durante a estação de cultivo de 2011, em todo o distrito de irrigação (B-XII) foi de $58168,8 \mathrm{hm}^{3}$, assim distribuídos em cada setor: $22154,7 \mathrm{hm}^{3}$ (Setor A), 21597,3 $\mathrm{hm}^{3}$ (Setor B) e 14416,8 $\mathrm{hm}^{3}$ (Setor C). O volume total aplicado seguiu a mesma ordem de área cultivada em cada setor. Ou seja, houve maior aplicação, em termos absolutos, no setor A que possui a maior 


\section{A. S. Andrade Junior et al.}

área cultivada (4974,3 ha), enquanto a menor aplicação ocorreu no setor C, com área cultivada de 3321,8 ha. Porém, avaliando-se a taxa de aplicação por área, obtida pela relação entre o volume total aplicado (hm3) (Tabela 5) e a área cultivada de cada setor (ha), observa-se que o setor B, apresentou a maior taxa de aplicação por área, com $5,721 \mathrm{hm}^{3} / \mathrm{ha}$ contra $4,454 \mathrm{hm}^{3} / \mathrm{ha}$ (Setor A) e $4,34 \mathrm{hm}^{3} / \mathrm{ha}$ (Setor C). Esse fato é particularmente importante, já que indica uma tendência dos irrigantes do setor $\mathrm{B}$ adotarem o manejo de irrigação mais adequado as necessidades hídricas dos cultivos, ou seja, sem a estratégia de aplicação de déficit hídrico. Outro fator que contribuiu para essa situação, é que no Setor $\mathrm{B}$, há o maior número de parcelas e área ocupada com o cultivo do tomate, irrigado por gotejamento, com as laterais por embaixo das fileiras de plantas, e manejado sempre com o solo próximo à capacidade de campo. Essa tendência foi confirmada posteriormente por declaração verbal do gerente do distrito, por ocasião de uma visita técnica ao B-XII.

Houve uma maior concentração da aplicação de água no período de junho a agosto, com 75,0\% (Setor A), 73,8\% (Setor B) e 72,7\% (Setor C) do volume total anual aplicado nesses períodos (Figura 2). Essa distribuição reflete a demanda evapotranspirativa da região, bem como do plano de cultivo em exploração na área no ano agrícola de 2011. O cultivo com maior área é o algodão, que é semeado a partir de segunda quinzena de maio e colhido na primeira quinzena de outubro. Como é um cultivo onde se adota um manejo de irrigação deficitário, a suspensão da irrigação, normalmente, ocorre com 30 dias de antecedência à colheita (primeira quinzena de setembro). Nesse mesmo período, há uma maior concentração da demanda evapotranspirativa da região (42,3\%) (Figura 2).

Considerando apenas os cultivos puros, que ocupam apenas 10,6\% da área do B-XII, os cultivos de tomate e milho foram os que apresentaram maiores volumes aplicados por unidade de área, com médias de $8862 \mathrm{~m}^{3} / \mathrm{ha} \mathrm{e} 6867 \mathrm{~m}^{3} /$ ha, respectivamente. Para o trigo, como é um cultivo de inverno, foram registrados os menores volumes aplicados por unidade de área, com média geral de apenas 1730 $\mathrm{m}^{3} /$ ha. Essa mesma tendência e volumes aplicados aproximados a esses foram relatados por Lorite et al. (2004b), em estudo realizado no Distrito de Irrigação Genil-Cabra, em Córdoba, região com características climáticas semelhantes a Lebrija. No setor B, foram registradas as maiores taxas de aplicação para todos os cultivos, e em especial, para o tomate $\left(9031 \mathrm{~m}^{3} / \mathrm{ha}\right)$ e o milho $\left(7049 \mathrm{~m}^{3} / \mathrm{ha}\right)$. Esse comportamento é devido ao fato dos irrigantes desse setor aplicar água com um manejo não deficitário e mais adequado as necessidades hídricas dos cultivos, além de que há uma maior predominância de área cultivada com tomate, em comparação com os demais setores.

\section{Estimativa da evapotranspiração dos cultivos}

Os valores de estimativa da ET dos cultivos obtidas com a aplicação do Kcb-Savi e Kcs são apresentados na Figura 3. A ETc média por setor, adotando o Kcb-Savi, foi de $5670 \mathrm{~m}^{3} / \mathrm{ha}(\mathrm{A}), 5897$ $\mathrm{m}^{3} / \mathrm{ha}$ (B) e $5882 \mathrm{~m}^{3} / \mathrm{ha} \mathrm{(C)} \mathrm{e} \mathrm{de} 5701 \mathrm{~m}^{3} / \mathrm{ha}(\mathrm{A}), 5917 \mathrm{~m}^{3} / \mathrm{ha}(\mathrm{B})$ e $5923 \mathrm{~m}^{3} / \mathrm{ha} \mathrm{(C),} \mathrm{com} \mathrm{o} \mathrm{uso} \mathrm{do} \mathrm{Kcs.}$ Os valores de ETc com o uso do Kcb-Savi e Kcs foram muito semelhantes e bem ajustados em todos os setores e no BXII, em ambas as formas de avaliação. Os percentuais de variação foram da ordem de $0,53 \%$ (A), $0,34 \%$ (B), $0,70 \%$ (C) e $0,52 \%$ (B-XII). As diferenças de estimativa ocorrem unicamente pelos desvios inerentes aos dois métodos de estimativa. Logicamente, como houve um bom ajuste entre os dois métodos de estimativa de ET, não há diferenças importantes na estimativa de ET nos três setores e no B-XII como um todo. Esse comportamento indica que a simplificação da estimativa do Kcs é adequada e substitui a contento o uso do modelo Kcb-Savi, conforme demonstrado em Mateos et al. (2013) e Gonzalez-Dugo et al. (2013).

\section{$\underline{\text { Índices de eficiência da irrigação }}$}

Os valores dos índices de eficiência de irrigação são apresentados na Figura 4. Para o índice RET, como a estimativa do índice é baseada no mesmo plano de cultivo, a ET potencial dos cultivos é a mesma nas duas formas de avaliação. As mínimas diferenças observadas devem-se unicamente as mínimas diferenças observadas na estimativa de ET usando os modelos Kcb-Savi e Kcs (Figura 3). Como essa diferença é mínima, os valores de RET variam muito pouco entre os dois métodos de estimativa de ETc. Em termos médios, em todo o B-XII, a variação do RET, entre os dois métodos de estimativa de ETc, foi de apenas 0,58\% (RET = 0,778, com o uso de Kcb-Savi, e RET = 0,783, com o 
uso do Kcs). Ou seja, a aplicação de água ao B-XII, no ano agrícola de 2011, permitiu que a ETc dos cultivos fosse atendida em $78 \%$ de sua evapotranspiração potencial.

Por seu conceito, o CDH varia acompanhando as variações observadas no RET. Como não houve alterações sensíveis no RET, logicamente as variações no $\mathrm{CDH}$ seguem a mesma tendência. A diferença média obtida foi de apenas 2,0\%, em todo o B-XII (CDH = 0,222, com o uso de Kcb-Savi, e $\mathrm{CDH}=0,217$, com o uso do Kcs). Logicamente, como o atendimento da ET dos cultivos foi de 78\%, a deficiência hídrica observada na área foi de $22 \%$, valor esse que pode ser considerado até como reduzido, tendo em conta que o algodão ocupa $49,2 \%$ da área e é manejado com irrigação deficitária.

No caso do ICUC, considerando-se a pequena variabilidade nos valores de ET dos cultivos (Figura 3) e que os volumes de água aplicados às parcelas não variam, já que se trata do mesmo plano de cultivo, a análise da variabilidade desse índice depende da variação observada nos outros dois parâmetros envolvidos na estimativa de ICUC, quais sejam, a precipitação efetiva e a variação no armazenamento de água no solo.

No caso da precipitação efetiva, foi calculada como a diferença entre a precipitação registrada menos a percolação profunda e o escoamento superficial. No método de estimativa da ET com o Kcs, usou-se os mesmos valores desses parâmetros obtidos dos balanços hídricos com o emprego do KcbSavi. Portanto, esse parâmetro não se constitui em fonte de variação para o comportamento do ICUC. Por isso, essa variabilidade se restringe apenas à variação ocorrida no armazenamento de água no solo. Como no método empregando o Kcs, usou-se para simplificar o cálculo, a variação média do armazenamento de água no solo advinda do emprego do método do Kcb-Savi (15 mm), logicamente, o ICUC variou, em cada setor e no B-XII, em função do quanto variou o armazenamento real de água no solo com relação a média. Observa-se que, o emprego da variação média do armazenamento de água no solo elevou o ICUC em 12,41\%, no B-XII, de maneira uniforme em todos os setores $(\mathrm{A}=12,96 \%$, $\mathrm{B}=11,95 \%$ e $\mathrm{C}=12,31 \%)$.

Conceitualmente, o valor do ICUC deve ser, no máximo, igual à unidade (ICUC $\leq 1)$, já que a ET dos cultivos não pode superar o volume efetivamente aplicado pela irrigação (Bos et al., 2005). Porém, observa-se que, o conceito teórico não foi obedecido nos setores A $(1,12)$ e $\mathrm{C}(1,14)$. Isso foi devido, provavelmente, ao fato de que o algodão, como um cultivo com manejo de irrigação deficitário, notadamente, na fase final de seu ciclo de cultivo, ocupa área considerável desses setores, e não teve sua ETc estimada de forma adequada pelo uso do Kcb-Savi. Ou seja, a ET do algodão foi superestimada em relação a ET real ocorrida em campo, o que levou o ICUC ser superior à unidade.

O índice Savi, por ser um índice que reflete as condições de crescimento vegetativo, indica, de forma indireta, o nível de estresse hídrico no solo ao qual o cultivo está submetido (Gonzalez-Dugo \& Mateos, 2008; Glenn et al., 2011), ou seja, não é um índice sensível para estimar a ET dos cultivos em condição de estresse hídrico no solo. No caso do setor B, apesar do cultivo do algodão ocupar quase a mesma área irrigada dos setores A e C $(48,9 \%)$, o ICUC foi inferior à 1 , resultado esse devido, notadamente, à elevada área ocupada com tomate (15\%). Conforme ressaltado anteriormente, os produtores de tomate manejam a irrigação sem déficit e com o solo sempre próximo à capacidade de campo, o que possibilita um elevado uso consumptivo da água de irrigação nesse setor.

Quanto ao RIS, como os volumes de água aplicados as parcelas e os volumes potenciais foram os mesmos, os valores de RIS são rigorosamente os mesmos com o emprego do Kcb-Savi e Kcs. O valor ideal para o RIS seria igual à 1 , onde se teria a igualdade entre o volume de água efetivamente aplicado e o volume teórico que potencializa a ETc (irrigação potencial). Nos setores A e C, com valores de RIS iguais a 0,951 e 0,938, respectivamente, obteve-se valores de RIS inferiores a 1 , indicando que efetivamente foi aplicado $95,1 \%$ e $93,8 \%$ do volume de água ideal para os cultivos. Esse comportamento foi devido à adoção da irrigação com déficit no cultivo do algodão, que ocupa área representativa nesses setores (Lorite et al., 2004a). Por outro lado, no setor B, com RIS igual a 1,19, significa que foi aplicado $19 \%$ a mais de água do que a teoricamente necessária para máxima ET dos cultivos. Novamente, a adoção do manejo de irrigação do tomate, com a manutenção de elevados conteúdos de água no solo, afetou o comportamento desse índice.

A estimativa do RWS avalia a contribuição de todo o volume de água aplicado (chuva mais irrigação) para o processo evapotranspirativo dos cultivos (Lorite et al., 2004a). No caso do B-XII, obteve-se RWS igual a 0,944, redução essa devido à contribuição da precipitação para atendimento de parte da ET dos cultivos. Logicamente, a contribuição da precipitação registrada durante o ciclo de cultivo do trigo, beterraba e girassol, típicos do período de inverno/primavera, foi responsável por essa 


\section{A. S. Andrade Junior et al.}

tendência. Esses valores de RWS são aproximados aos obtidos em outros estudos conduzidos por Kloezen \& Garces-Restrepo (1998), Molden et al. (1998), Burt \& Styles (1999) e Lorite et al. (2004a).

\section{$\underline{\text { Índices de produtividade da água }}$}

Na Tabela 2 são apresentados os dados relativos à produção e a evapotranspiração das parcelas com as quais se processou a estimativa do índice de produtividade da água de irrigação com base na água evapotranspirada pelos cultivos de algodão, beterraba e tomate. Essa estimativa foi efetuada com os dados de 580 parcelas, sendo 132 (setor A), 279 parcelas (setor B) e 169 (setor C). Considerando-se todo o B-XII, utilizou-se 112 parcelas de algodão (529,8 ha), 326 de beterraba (1329,5 ha) e 142 de tomate (441,3 ha) (Tabela 2).

Os valores de produtividade da água (WP) obtidos no B-XII situam-se dentro da faixa dos valores observados em outros distritos de irrigação apresentados por Molden et al. (1998). O cultivo do tomate foi o que apresentou melhor índice de produtividade da água de irrigação, com 18,049 $\mathrm{kg} / \mathrm{m}^{3}$ de água, seguido pela beterraba $\left(10,431 \mathrm{~kg} / \mathrm{m}^{3}\right)$ e o algodão $\left(0,660 \mathrm{~kg} / \mathrm{m}^{3}\right)$. Esse resultado é unicamente dependente da relação entre a produção total obtida em função do volume de água evapotranspirada pelo cultivo. O tomateiro é produzido com um manejo de irrigação não deficitário, com o solo sempre mantido próximo a capacidade de campo, resultando em elevadas produtividades, superiores a $100 \mathrm{t} / \mathrm{ha}$ (Tabela 2).

Por outro lado, o cultivo do algodão é manejado com irrigação deficitária, notadamente, em sua fase final de desenvolvimento, o que afeta a produção final da cultura. É um cultivo subsidiado, cujo sistema de produção é dirigido para garantir a obtenção de produtividades em torno de $3000 \mathrm{~kg} / \mathrm{ha}$, nível mínimo que garante o pagamento do subsidio por parte do governo espanhol. Por isso, o desempenho do índice de produtividade da água é tão reduzido.

Quanto à beterraba, o desempenho do índice de produtividade da água de irrigação é intermediário. Como é cultivo típico de inverno/primavera, tem parte de sua necessidade hídrica atendida pela precipitação, o que permite alcançar níveis de produtividade elevados $(\approx 70 \mathrm{t} / \mathrm{ha}) \mathrm{sem}$ a necessidade de aplicação de irrigação plena.

Os resultados de WP obtidos para os cultivos do algodão e beterraba variaram na faixa de valores obtidos por Gonzalez-Dugo \& Mateos (2008), em estudo conduzido na mesma área do B-XII, no ano agrícola de 2008. Os autores obtiveram WP de 0,3 a $0,78 \mathrm{~kg} / \mathrm{m}^{3}$ (algodão) e de 7,15 a 14,8 $\mathrm{kg} / \mathrm{m}^{3}$ (beterraba). Concluíram que, o suprimento hídrico limitou apenas parcialmente a produtividade da beterraba, já que outros fatores ambientais e de produção, normalmente, afetam bem mais a produtividade da cultura. Por outro lado, o manejo de irrigação deficitário aplicado ao cultivo do algodão foi a principal causa do seu baixo rendimento produtivo.

\section{CONCLUSÕES}

a) O distrito de irrigação "Sector B-XII del Bajo Guadalquivir" apresenta índices de eficiência de aplicação de água satisfatórios, com valores de RET $=0,778, \mathrm{CDH}=0,222, \mathrm{ICUC}=0,938, \mathrm{RIS}=$ 1,012 e RWS $=0,935$, com o emprego do Kcb-Savi. A adoção do Kcs possibilita a estimativa dos índices de eficiência sem diferenças significativas em relação ao emprego do Kcb-Savi (RET = 0,783, $\mathrm{CDH}=0,217$, ICUC $=1,037$, RIS $=1,028$ e RWS $=0,944$ ).

b) $\mathrm{O}$ cultivo do tomate $\left(18,05 \mathrm{~kg} / \mathrm{m}^{3}\right)$ apresenta produtividade da água de irrigação superior aos cultivos do algodão $\left(0,66 \mathrm{~kg} / \mathrm{m}^{3}\right)$ e beterraba $\left(10,43 \mathrm{~kg} / \mathrm{m}^{3}\right)$, reflexo do adequado manejo de água de irrigação adotado pelos tomaticultores.

\section{REFERENCIAS BIBLIOGRÁFICAS}

ADLER-GOLDEN, S.M., MATTHEW, M.W., BERNSTEIN, L.S., LEVINE, R.Y., BERK, A., RICHTSMEIER, S.C., ACHARYA, P.K., ANDERSON, G.P., FELD, G., GARDNER, J., HOKE, M., JEONG, L.S., PUKALL, B., RATKOWSKI , A., BURKE, H.K. "Atmospheric Correction for Short-wave Spectral Imagery Based on MODTRAN4," Summaries of the Eighth Annual JPL Earth Science Workshop, Vol. I, 1999, available at http://makalu.jpl.nasa.gov. 
ALLEN, R.G., PEREIRA, L.S., RAES, D., SMITH, M. Crop evapotranspiration: guidelines for computing crop water requirements. Rome: FAO, 300p. 1998. (FAO Irrigation and Drainage Paper, 56).

BAUSCH, W.C., NEALE, C.M.U. Crop coefficients derived from reflected canopy radiation: a

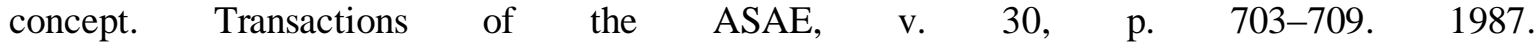
http://dx.doi.org/10.13031/2013.30463

BERK, A., L. S., ANDERSON, G. P. ANDERSON, P. K. ACHARYA, D. C. ROBERTSON, J .H. CHETWYND, S. M. ADLER-GOLDEN. "MODTRAN Cloud and Multiple Scattering Upgrades with Application to AVIRIS," Remote Sens. Environ. 65 , 367-375. 1998. http://dx.doi.org/10.1016/S0034-4257(98)00045-5

BOS, M.G, BURTON, M.A., MOLDEN, D.J. Irrigation and drainage performance assessment practical guidelines. CABI Publishing, Wallingford, UK, 2005. 158 pp.

BULLER, O., MANGES, H.L., STONE, L.R., WILLIAMS, R. Modeled crop water use and soil water drainage. Agricultural Water Management, v. 19, p. 117-134. 1991. http://dx.doi.org/10.1016/0378-3774(91)90003-2

BURT, C.M., STYLES, S.W. Modern water control and management practices in irrigation. Impact on performance. Rome: FAO, 1999. 286p. (Water Reports, 19).

CALERA, A., GONZALEZ-PIQUERAS, J., MELIA, J. Monitoring barley and corn growth from remote sensing data at field scale. International Journal of Remote Sensing, v. 25, p. 97-109. 2004. http://dx.doi.org/10.1080/0143116031000115319

CAMPOS, I., NEALE, C.M.U., CALERA, A., BALBONTIN, C., GONZÁLEZ-PIQUERAS, J. Assessing satellite-based basal crop coefficients for irrigated grapes (Vitis vinifera L.). Agricultural Water Management, v. 97, p. 1760-1768. 2010.

ESRI. ENVIRONMENTAL SYSTEMS RESEARCH INSTITUTE, ArcGIS Professional GIS for the desktop, version 9.3. Software. 2008.

FAO. Agriculture and water scarcity: a programmatic approach to water use efficiency and agricultural productivity. Twentieth Session, Committee on Agriculture, COAG/2007/7. Rome. 2007.

GLENN, E.P., NEALE, C.M.U., HUNSAKER, D.J., NAGLER, P.L. Vegetation index-based crop coefficients to estimate evapotranspiration by remote sensing in agricultural and natural ecosystems. Hydrological Processes, v. 25, p. 4050-4062. 2011. http://dx.doi.org/10.1002/hyp.8392

GONZALEZ-DUGO, M. P., NEALE, C. M. U., MATEOS, L., KUSTAS, W. P., PRUEGER, J., ANDERSON, M. C., LI, F.: A comparison of operacional remote-sensing-based models for estimating crop evapotranspiration, Agr. Forest Meteorol., 149, 1843-1853, 2009. http://dx.doi.org/10.1016/j.agrformet.2009.06.012

GONZALEZ-DUGO, ESCUIN, S., CANO, F., CIFUENTES, V., PADILLA, F.L.M., TIRADO, J.L., OYONARTE, N., FERNANDEZ. P., MATEOS, L. Monitoring evapotranspiration of irrigated crops using crop coefficients derived from time series of satellite images. II. Application on basin scale. Agricultural Water Management, v. 125, p. 92-104. 2013. http://dx.doi.org/10.1016/j.agwat.2013.03.024

GONZÁLEZ-DUGO, M.P., L. MATEOS. Spectral vegetation indices for benchmarking water productivity of irrigated cotton and sugarbeet crops. Agricultural Water Management 95:48-58. 2008. http://dx.doi.org/10.1016/j.agwat.2007.09.001

HEINEMANN, A.B., HOOGENBOOM, G., FARIA, R.T. Determination of spatial water requirements at county and regional levels using crop models and GIS. An example for the State of Parana, Brazil. Agricultural Water Management, v. 52, p. 177-196. 2002. http://dx.doi.org/10.1016/S0378-3774(01)00137-8

HUETE, A. R.: A soil-adjusted vegetation index (SAVI), Remote Sens, Environ., 25, 295-309, 1988. http://dx.doi.org/10.1016/0034-4257(88)90106-X

ITT Industries Inc., ENVI (Environment for Visualizing Images) Version 4.7. ITT Industries Inc., Boulder, US. 2009.

KLOEZEN, W.H, GARCES-RESTREPO, C. Assessing irrigation performance with comparative indicators: the case of the Alto Rio Lerma irrigation district, Mexico. Colombo, Sri Lanka: Irrigation Water Management Institute, 1998. 39p. (Research Report , 22). 
LORITE, I.J., MATEOS, L., FERRERES, E. Evaluating irrigation performance in a Mediterranean environment. I. Model and general assessment of an irrigation scheme. Irrigation Science, v. 23, p. 77-84, 2004a. http://dx.doi.org/10.1007/s00271-004-0095-9

LORITE, I.J., MATEOS, L., FERRERES, E. Evaluating irrigation performance in a Mediterranean environment. II. Variability among crops and farmers. Irrigation Science, v. 23, p. 85-92, 2004b. http://dx.doi.org/10.1007/s00271-004-0096-8

LORITE, I.J., MATEOS, L., ORGAZ, F., FERRERES, E. Assessing deficit irrigation strategies at the level of an irrigation district. Agricultural Water Management, v. 91, p. 51-60. 2007.

LOZANO, D., MATEOS, L. Usefulness and limitations of decision support systems for improving irrigation scheme management. Agricultural Water Management 95, 409-418, 2008. http://dx.doi.org/10.1016/j.agwat.2007.11.003

MATEOS, L., GONZALEZ-DUGO, M.P., TESTI, L., VILLALOBOS, F.J. Monitoring evapotranspiration of irrigated crops using crop coefficients derived from time series of satellite images. I. Method validation. Agricultural Water Management, v. 125, p. 81-91. 2013. http://dx.doi.org/10.1016/i.agwat.2012.11.005

MIMAM - Ministerio de Medio Ambiente. Plan hidrológico nacional. Madrid. 2000.

MOLDEN, D.J., SAKTHIVADIVEL, R., PERRY, C.J., FRAITURE, C. de, KLOEZEN, W.H. Indicators for comparing performance of irrigated agricultural systems. Colombo, Sri Lanka: Irrigation Water Management Institute, 1998. 26p. (Research Report, 20).

NEALE, C.M.U., BAUSCH, W.C., HEERMAN, D. F. Development of reflectance-based crop coefficients for corn. Transactions of the ASAE, 32(4), 1891-1899, 1989.

ROUSE, J.W., HAAS, R.H., SCHELL, J.A., DEERING, D.W., HARLAN, J.C. Monitoring the vernal advancement and retrogradation of natural vegetation. NASA/GSFC, Type III, Final report, Greenbelt MD, 1-371. 1974.

VERMOTE, E.F., N. El Saleous, C.O. Justice, Y.J. Kaufman, J.L. Privette, L. Remer, J.C. Roger and D. Tanre. "Atmospheric Correction of Visible to Middle-Infrared EOS-MODIS Data Over Land Surfaces: Background, Operational Algorithm and Validation," J. Geophys. Res., Vol. 102, pp. 17131-17141.1997.

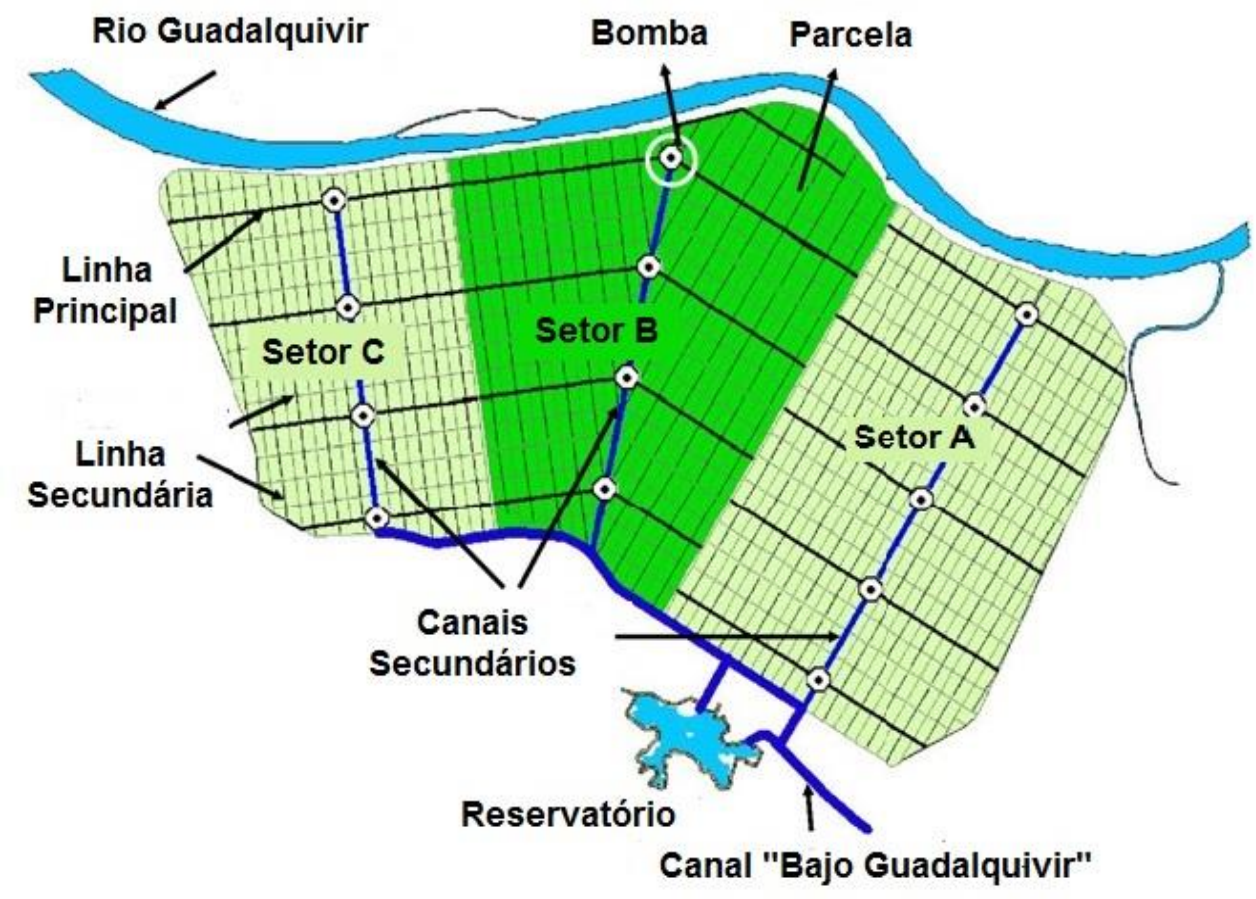

Figura 1. Esquema de distribuição de água do distrito de irrigação "Sector B-XII del Bajo Guadalquivir". Adaptado de Lozano \& Mateos (2008). 
Tabela 1. Volumes mensais de água aplicados $\left(\mathrm{hm}^{3}\right)$ em cada setor do distrito de irrigação "Sector BXII del Bajo Guadalquivir" durante o período de janeiro a setembro de 2011.

\begin{tabular}{crrlccccccc}
\hline Setor & \multicolumn{1}{c}{ Jan } & \multicolumn{1}{c}{ Fev } & Mar & Abr & Mai & Jun & Jul & Ago & Set & Total \\
\hline A & 9,4 & 145,9 & 192,8 & 2252,6 & 2753,6 & 5155,3 & 6396,7 & 5110,9 & 137,6 & 22154,7 \\
B & 9,8 & 24,9 & 420,9 & 2392,1 & 2783,2 & 5491,4 & 5797,4 & 4527,8 & 149,8 & 21597,3 \\
C & 0,0 & 11,8 & 305,1 & 1546,6 & 1967,4 & 3324,4 & 3743,2 & 3387,8 & 130,5 & 14416,8 \\
\hline Total & 19,2 & 182,6 & 918,8 & 6191,3 & 7504,2 & 13971,1 & 15937,2 & 13026,5 & 417,8 & 58168,8 \\
\hline
\end{tabular}

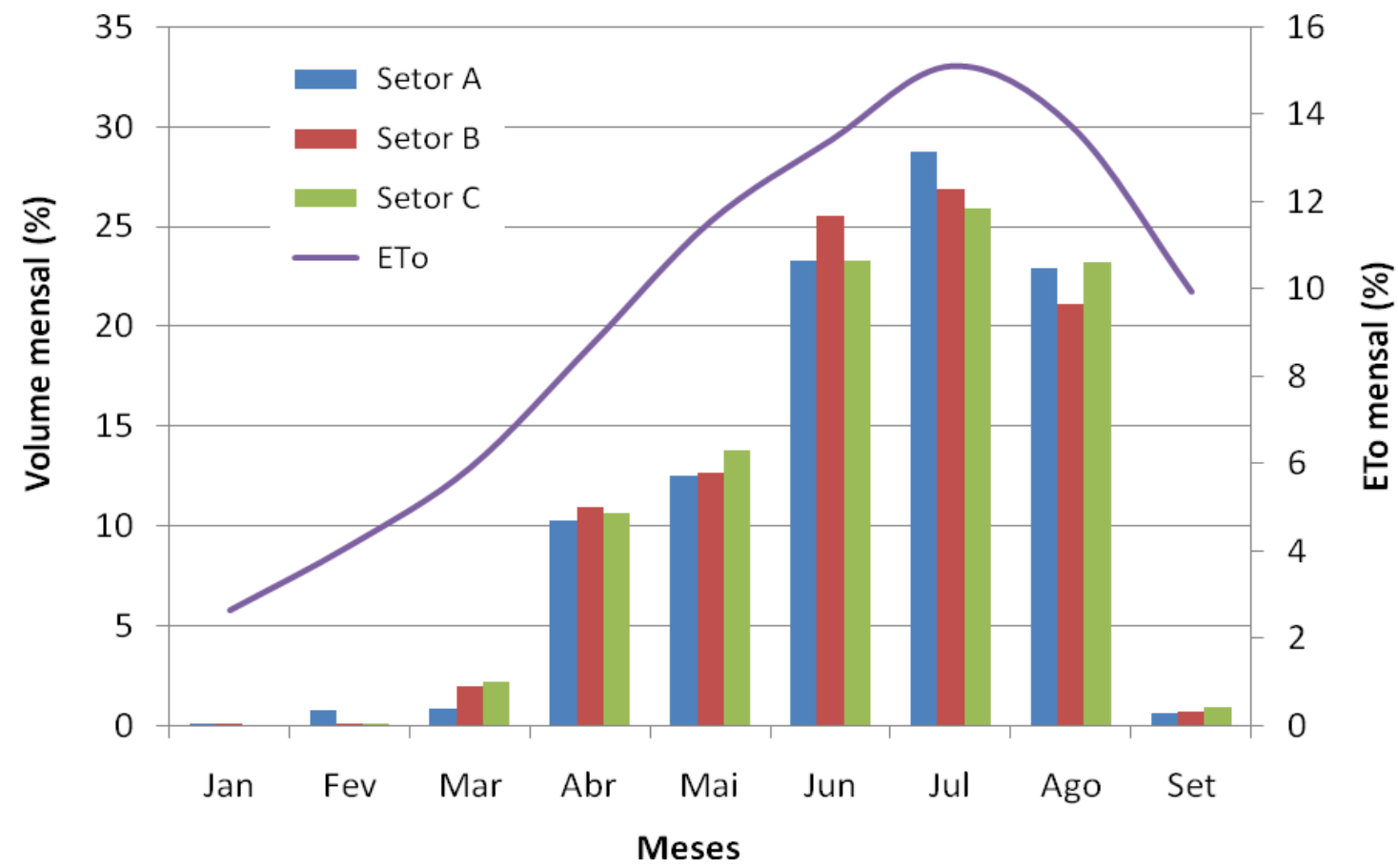

Figura 2. Distribuição percentual mensal do volume total aplicado e da evapotranspiração de referencia (ETo) durante o período de janeiro a setembro de 2011.

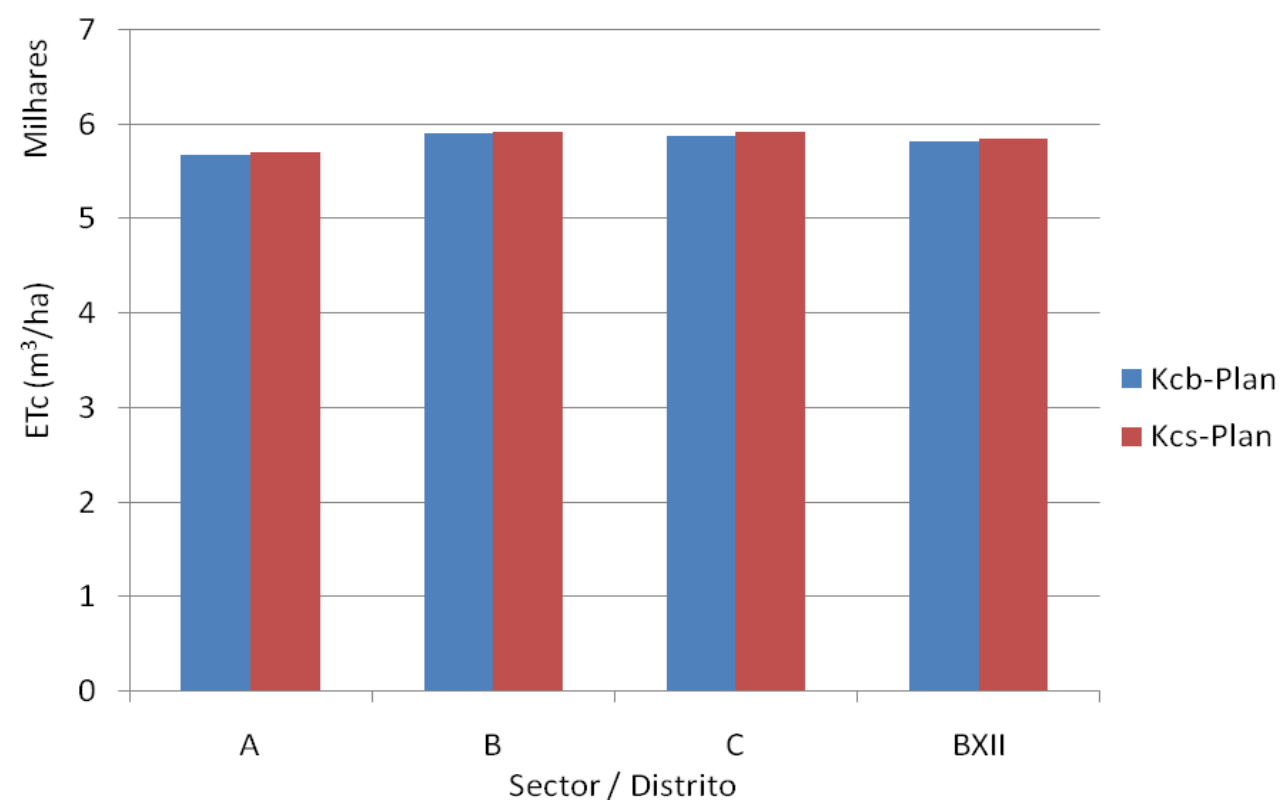

Figura 3. Evapotranspiração dos cultivos avaliados obtida com a aplicação de Kcb-Savi (Kcb-Plan) e Kcs (Kcs-Plan), por setor/distrito, com o plano de cultivos da comunidade dos irrigantes. 

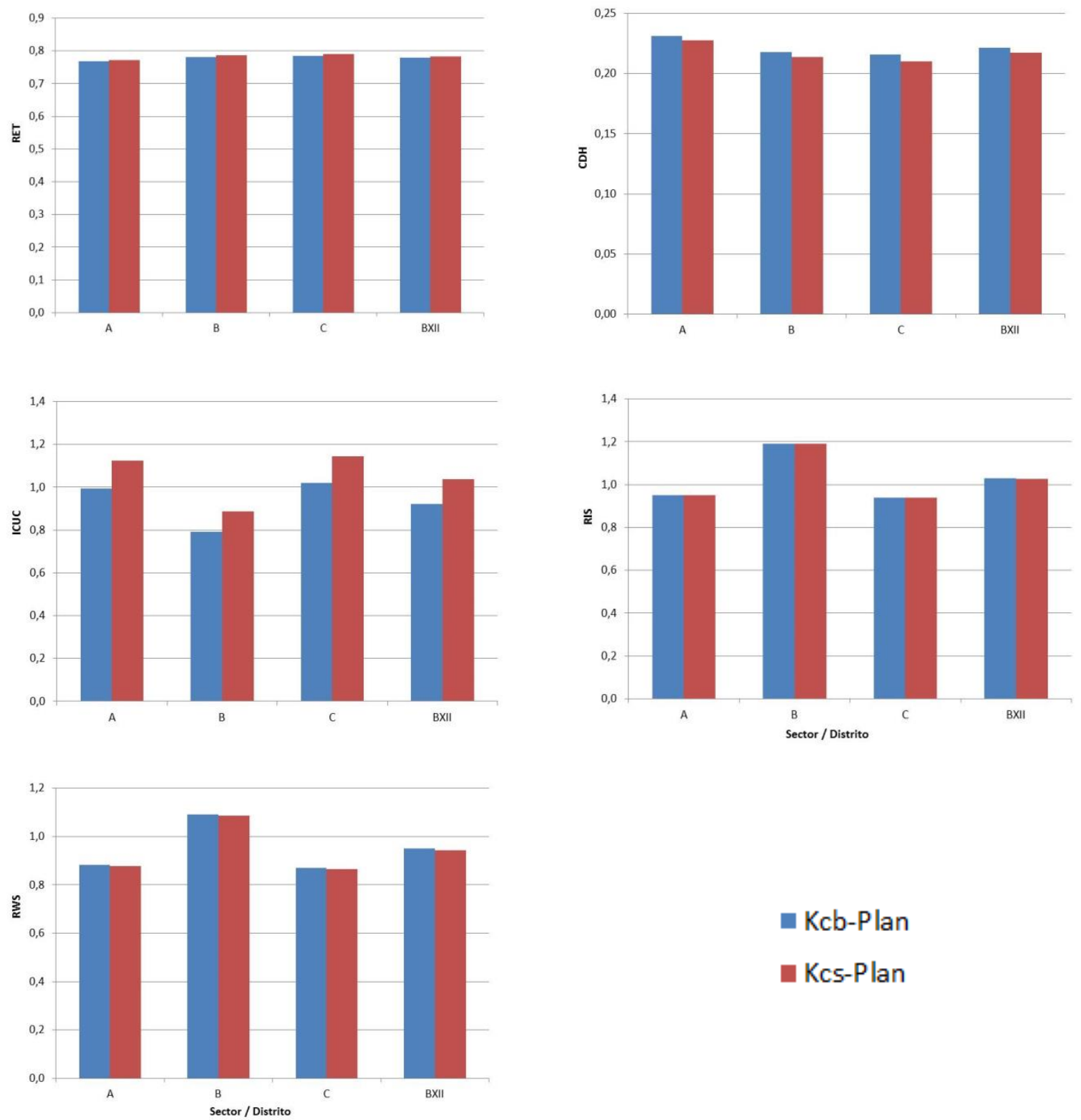

\section{- Kcb-Plan \\ Kcs-Plan}

Figura 4. Índices de eficiência de irrigação obtidos com a simulação de ETc com Kcb-Savi (Kcb-Plan e Kcs (Kcs-Plan), ambos com base no plano de cultivo da comunidade dos irrigantes.

Tabela 2. Número de parcelas (NP), área (ha), produção (t), produtividade (t/ha), evapotranspiração (ETc) $\left(\mathrm{m}^{3} / \mathrm{ha}\right)$ e índice de produtividade da água (WP) $\left(\mathrm{kg} / \mathrm{m}^{3}\right)$, para os cultivos de algodão, beterraba e tomate, no ano agrícola de 2011.

\begin{tabular}{lcrrcrr}
\hline Cultivos & NP & \multicolumn{1}{c}{$\begin{array}{c}\text { Área } \\
(\text { ha })\end{array}$} & $\begin{array}{c}\text { Produção } \\
(\mathrm{t})\end{array}$ & $\begin{array}{c}\text { Produtividade } \\
(\mathrm{t} / \mathrm{ha})\end{array}$ & $\begin{array}{c}\text { ETc } \\
\left(\mathrm{m}^{3} / \mathrm{ha}\right)\end{array}$ & $\begin{array}{r}\text { WP } \\
\left(\mathrm{kg} / \mathrm{m}^{3}\right)\end{array}$ \\
\hline Algodão & 112 & 529,79 & 2023,17 & 3,66 & 5558,72 & 0,660 \\
Beterraba & 326 & 1329,54 & 96765,90 & 71,98 & 6895,57 & 10,431 \\
Tomates & 142 & 441,26 & 45603,51 & 102,94 & 5714,84 & 18,049 \\
\hline Total & 580 & 2300,59 & 144392,57 & & & \\
\hline
\end{tabular}

